Service social

\title{
Les effets des interventions auprès des personnes silencieuses dans les groupes
}

\section{Chantal Leclerc et Patricia Paquet}

Volume 46, numéro 1, 1997

Politique et intervention

URI : https://id.erudit.org/iderudit/706750ar

DOI : https://doi.org/10.7202/706750ar

Aller au sommaire du numéro

Éditeur(s)

École de service social de l'Université Laval

ISSN

1708-1734 (numérique)

Découvrir la revue

Citer cet article

Leclerc, C. \& Paquet, P. (1997). Les effets des interventions auprès des personnes silencieuses dans les groupes. Service social, 46(1), 67-94. https://doi.org/10.7202/706750ar
Résumé de l'article

Un phénomène fréquemment observable dans les groupes est la distribution inégale du temps de parole entre les membres. En écho à cette situation, plusieurs stratégies d'intervention sont proposées pour inciter les personnes silencieuses à affirmer leur position. Ces stratégies sont en accord avec les représentations généralement négatives qu'on se fait du silence dans les milieux des services sociaux et de l'éducation. Après une description de quatre représentations dominantes, l'article montre que le silence n'a pas à être réduit à un mode atrophié ou dévié d'expression, c'est-à-dire un mode à corriger à tout prix. Il montre aussi que les interventions où l'on cherche à faire parler les personnes silencieuses, si bien intentionnées soient-elles, ont parfois un effet contraire à celui recherché. 


\section{Les effets des interventions auprès des personnes silencieuses dans les groupes}

Chantal LECLERC

Patricia PAQUET

Un phénomène fréquemment observable dans les groupes est la distribution inégale du temps de parole entre les membres. En écho à cette situation, plusieurs stratégies d'intervention sont proposées pour inciter les personnes silencieuses à affirmer leur position. Ces stratégies sont en accord avec les représentations généralement négatives qu'on se fait du silence dans les milieux des services sociaux et de l'éducation. Après une description de quatre représentations dominantes, l'article montre que le silence n'a pas à être réduit à un mode atrophié ou dévié d'expression, c'està-dire un mode à corriger à tout prix. II montre aussi que les interventions où l'on cherche à faire parler les personnes silencieuses, si bien intentionnées soient-elles, ont parfois un effet contraire à celui recherché.

A phenomenon which occurs commonly within groups is the uneven distribution of speach time between members. In answer to this situation, several intervention strategies are proposed in order to incite quiet individuals to assert their opinion. These strategies agree with the negative representation of silence which is common in the fields of education and social services. After describing four major representations of silence, this article shows that silence does not have to be assimilated with a weakened or otherwise deviated means of expression, i.e. something to be corrected at any cost. It also shows that interventions aimed at forcing quiet individuals to speak, as well-meaning as they may be, sometimes yield results contrary to what was intended. 
Un des phénomènes les plus facilement observables dans la plupart des groupes est la distribution inégale du temps de parole entre les membres : chaque groupe comprend généralement des personnes qui s'expriment beaucoup et des personnes qui sont reconnues, voire étiquetées, comme silencieuses. Bien que les stratégies d'intervention élaborées en animation de groupe soient généralement appropriées pour équilibrer la participation des membres dans un groupe, il convient de s'interroger sur les effets pervers de certaines de ces stratégies. Aussi bien intentionnées et apparemment justifiées soient-elles, les interventions qui visent à aider un membre silencieux à s'exprimer ont parfois un effet contraire à celui recherché : plus on lui tend des perches, plus il se retire et se replie.

Une tendance actuellement dominante en intervention psychosociale auprès des groupes est de considérer le silence comme l'indice d'une difficulté à surmonter, sinon comme un problème à résoudre. En proposant d'y voir plutôt un mode complet d'expression, nous visons à mettre en lumière les pièges et les inconvénients de l'image négative et appauvrie qu'on se fait généralement du silence dans les sociétés occidentales en général et, plus spécifiquement, dans les milieux de l'éducation et du travail social.

Afin de montrer que le silence n'a pas à être réduit à un mode secondaire, atrophié ou dévié d'expression, c'est-à-dire un mode à corriger ou à améliorer, l'article met d'abord en lumière la richesse polysémique du silence et la relativité des interprétations qu'on en donne selon les personnes, selon les situations et selon les milieux ethnoculturels dans lesquels il s'inscrit. Par la suite, quatre représentations du silence, dominantes dans les milieux des services sociaux et de l'éducation, sont dégagées, soit le silence vu comme signe de résistance, comme indice de souffrance psychologique, comme une offense aux autres et comme réaction à l'oppression. L'article se poursuit par une discussion des effets pervers susceptibles d'être produits par les interventions qui se dégagent de ces représentations négatives du silence. II se termine par une analyse des coûts et bénéfices du silence tels qu'ils peuvent être vécus par les personnes silencieuses, par les autres membres plus loquaces et par les intervenants ou intervenantes. En conclusion, certaines situations de silence qui justifient une intervention sont identifiées et 
quelques pistes d'intervention sont proposées aux intervenantes et intervenants.

\section{UN MODE COMPLET D'EXPRESSION}

À première vue, le silence peut paraître bien simple à définir. Spontanément, on dira qu'il s'agit d'un vide entre les sons ou d'une absence de sons d'une durée variable. Cependant, lorsqu'on s'y arrête plus longuement, on se rend compte que ce n'est pas le vide ou l'absence de sons "en soi » qui intéresse les intervenants sociaux, mais plutôt l'absence de "quelque chose " et, plus précisément, l'absence de certains sons particuliers qu'on s'attend à entendre (Bilmes, 1994). En fait, chaque situation sociale fait naître certaines attentes particulières et, plus spécifiquement, certains sons qui par leur absence même portent un message. Ce n'est que parce que certaines personnes, dans une situation donnée, anticipent "quelque chose » que cette " absence » peut être notée, ressentie et jugée pertinente ou significative. Par opposition au " silence absolu », ces " silences sociaux ${ }^{1}$ » sont donc indissociables des sujets qui les interprètent; ils sont aussi indissociables des sons qui les précèdent ou qui les suivent, c'est-à-dire du contexte d'interaction qui procure aux partenaires des repères à partir desquels chacun règle sa conduite.

Pour comprendre les silences, il convient donc de se référer aux théories de la communication qui reconnaissent que les échanges se font toujours à deux niveaux.

La sémiologie structurale et la théorie classique de la communication, décrites par Sfez (1992), distinguent: Ia dimension instrumentale et codée de la communication, d'une part; et la dimension relationnelle et expressive, d'autre part. La première dimension s'associe à la "dénotation " et la seconde à la "connotation ». Dans les termes de la théorie de la communication développée par les chercheurs de l'école de Palo Alto (Watzlawick, Helmick-Beavin et Jackson, 1972), on parle plutôt: du " contenu » (instrumental) qui correspond au "langage digital ", la plupart du temps verbal; et de la "relation » qui correspond au «langage analogique », très souvent non verbal (Leclerc, à paraître, p. 37).

1. Nous parlons des silences, au pluriel, parce qu'ils sont aussi nombreux que les situations locales et ponctuelles desquelles ils émergent. 
Le « contenu » des messages constitue donc le premier niveau de la communication et correspond au dénotatif. En soi et à ce niveau, le message ne prête pas à interprétation : les interlocuteurs s'échangent de l'information qu'ils encodent et décodent selon des conventions syntaxiques et lexicales précises.

Pourtant, à un second niveau, chaque message comporte une valeur ajoutée qui correspond à ce qu'on nomme la connotation (Sfez, 1992) ou, dans les termes de l'école de Palo Alto, à la dimension analogique du langage (Watzlawick, Helmick-Beavin et Jackson, 1972). Ce niveau renvoie davantage à la manière dont le message doit être entendu et à la « relation » que les interlocuteurs cherchent à établir ensemble. Les intonations de la voix, les hésitations, les gestes, le contexte, l'histoire qui lie les interlocuteurs, leur mimique se combineront pour aider à interpréter le sens de ce message.

Les silences se situent donc d'emblée dans la sphère du langage connotatif. Si l'on peut saisir assez précisément le sens de certains messages par une simple activité de décodage, en se fondant sur l'usage conventionné des règles syntaxiques et lexicales d'une langue, chaque silence doit plutôt s'apprivoiser et s'interpréter dans les rapports entre les personnes. C'est précisément cette équivocité irréductible qui fait la richesse des silences. Loin d'être une absence de communication, le silence est un mode privilégié, raffiné et incontournable de communication (Lewis, 1977), une forme particulière de langage qui permet d'exprimer des choses inexprimables par les mots. Bilmes (1994) précise que le silence, dans la conversation, n'est pas " rien »; il n'est pas non plus un « manque » ou encore l'opposé de la communication. II fait partie intégrante du processus de production de sens de la conversation.

\section{LES ASPECTS POSITIFS DU SILENCE}

Même si ce n'est pas une perception généralisée dans les sociétés occidentales, le silence peut être vécu d'une manière très positive et perçu comme un élément essentiel de la communication et de l'apprentissage. Dans une communication pleine, celui qui écoute doit garder le silence, extérieurement et intérieurement, pour être en mesure de bien recevoir et comprendre le message (Fages, 1990). Celui qui s'exprime doit laisser du temps 
passer entre ses messages pour observer et prendre en considération les effets qu'il suscite (Richard, 1995).

Si certaines personnes subissent leur propre silence avec des sentiments plus ou moins intenses d'anxiété, il faut reconnaître que d'autres le vivent avec beaucoup de sérénité (du moins tant que les pressions extérieures ne les placent pas dans l'obligation de modifier leur conduite): elles choisissent délibérément de se taire parce qu'elles préfèrent nettement écouter ce que les autres ont à dire plutôt que de parler ellesmêmes (Lederman, 1982).

Sur le plan de l'apprentissage, le silence peut aussi être associé à un processus vicariant qui passe par l'écoute et l'observation actives des personnes qui disposent des savoirs et des savoir-faire qu'on veut imiter (Bandura, 1980). Plutôt que d'être vu comme un signe de passivité, le silence sera alors reconnu comme un mode privilégié d'apprentissage et d'intégration.

\section{L'ASPECT CULTUREL DU SILENCE}

Dans la culture nord-américaine, l'expression verbale est fortement valorisée comparativement au silence. La capacité de prendre la parole s'associe généralement à une socialisation qui encourage l'affirmation de soi, la spontanéité et la révélation de ses sentiments. À l'inverse, le silence est souvent connoté négativement, parce qu'on l'associe à la timidité, au manque de confiance en soi ou à la passivité. Sorentillo et Boutiller (1975, cités dans Guimond, 1994) ont démontré que la personne qui parle le plus dans un groupe (ce qui se mesure par le nombre d'interventions qu'elle fait) a beaucoup plus de chances d'être considérée comme compétente et influente, et cela, même si ses commentaires sont beaucoup moins pertinents et utiles, comparativement à ceux d'une autre personne peu loquace dont les commentaires sont de meilleure qualité.

Une exploration des différences qui existent d'une culture à une autre concernant la conception que les gens se font du silence permet de constater que ce qui semble aller de soi dans notre culture peut se vivre d'une façon radicalement différente ailleurs. Ainsi, chez les Asiatiques, il y a un dicton qui dit qu'une personne ne devrait parler que si la qualité de ce qu'elle a à dire est supérieure à la qualité du silence qu'elle rompt (Bilmes, 1994, p. 74). L'humilité, l'intériorité et la tranquillité associées au silence 
sont des signes de sagesse, tandis que le fait de s'exprimer beaucoup verbalement sera vu comme un manque de contrôle sur soi. Contrairement aux Nord-Américains qui valorisent la spontanéité, l'expression de soi et l'ouverture, ce sont plutôt les attitudes réservées qui seront considérées comme signes de raffinement, de distinction et de force intérieure (Ching et Prosen, 1980).

Yamaguchi étudie la compatibilité des traits culturels japonais avec la participation à des groupes d'entraide ou de psychothérapie. II souligne que les Japonais, vivant nombreux dans de petits espaces, ont été socialisés à éviter d'ouvrir et d'envenimer des conflits en privilégiant la passivité et la patience plutôt que l'affirmation et l'argumentation. C'est dans ce contexte appelé « kotage-senu-kuni », marqué par l'héritage de Confucius et du bouddhisme, que la communication non verbale s'est raffinée depuis des milliers d'années. Leur proverbe qui dit que "The nail that sticks up will be hammered down » (cité dans Yamaguchi, 1986, p. 576) traduit bien les normes implicites culturellement valorisées.

Les Finlandais ont eux aussi une vision très positive du silence. Ils apprécient les gens qui parlent peu autant qu'ils se méfient des flots de paroles (Lehtonen et Sajavaara, 1985). On dira dans ce pays que chacun doit soigneusement peser ses mots parce que la parole, une fois prononcée, ne peut être retirée : elle peut, à elle seule, allumer un feu impossible à éteindre par la suite. Plusieurs dictons populaires témoignent de ces valeurs ${ }^{2}$.

En accord avec ces traits culturels, les Finlandais sont capables de tolérer plus longtemps les silences dans les conversations que les gens d'Amérique ou d'Europe centrale. Même ces petits mots de rétroaction qu'on prononce souvent pour assurer l'autre de son écoute (oui, je vois, c'est bien, je comprends, etc.) sont jugés «intrusifs » chez les Finlandais. Ceux-ci s'expriment sans tolérer les interruptions; ils écoutent en silence et se contentent de hochements de tête, de mimiques et

2. Exemples de dictons populaires : « - II suffit d'un mot pour faire un tas de problèmes. - N'oublie pas que tu n'as qu'une bouche, mais deux oreilles. - Le chien qui aboie n'attrape pas son lièvre. - Un mot est aussi bon que neuf. " 
du contact visuel. Devant cette réserve verbale et ce type d'écoute bien étrangère à "l'écoute active à l'américaine », la personne issue d'une culture qui valorise l'extériorisation verbale pourra interpréter le silence finlandais comme un indice d'indifférence, d'ennui, de mauvaise humeur, voire d'hostilité.

Enfin, on accorde aussi une grande valeur au silence dans la culture autochtone. Collier (1993) rapporte à ce sujet que les élèves autochtones inscrits dans les cégeps se distinguent nettement des autres. Leur silence est tel qu'il ébranle les façons de faire des professeurs qui peuvent avoir l'impression qu'ils ne participent pas en classe, qu'ils ne comprennent pas ou qu'ils ne s'intéressent pas aux contenus d'enseignement. En fait, les élèves autochtones participent, mais d'une autre manière. Les modes d'apprentissage valorisés chez les autochtones sont, entre autres, l'observation et l'imitation de modèles. Cette femme autochtone à qui on demandait d'enseigner une pratique ancestrale de traitement des fourrures à des jeunes de sa communauté incarne bien cette spécificité culturelle lorsqu'elle répond que les jeunes qui veulent apprendre n'ont qu'à venir s'installer près d'elle pour l'observer. Dans les classes ordinaires, les jeunes autochtones considèrent que c'est le rôle du professeur de faire des démonstrations et de parler. Leur rôle, à eux, est d'écouter et d'être attentifs.

Ces quelques études sur les préférences et les habitudes de différents groupes culturels face au silence montrent bien que les attitudes interventionnistes envers les personnes silencieuses qu'on privilégie généralement dans les milieux de l'éducation et du travail social ne peuvent être considérées comme allant de soi. Elles méritent d'être discutées.

\section{LES SILENCES REPRÉSENTÉS COMME DES PROBLĖMES À RÉSOUDRE}

Les représentations sociales que les gens se font des phénomènes sont des ensembles organisés d'informations, d'opinions, de croyances et d'attitudes à propos d'un objet particulier ou d'une situation spécifiée (Monteil et Mailhot, 1988, p. 10). Elles font partie des systèmes collectifs de valeurs. Bien plus que de simples reflets de la réalité, les représentations sont des organisations signifiantes et des systèmes d'interprétation et d'anticipation de la réalité qui régissent les relations des individus à leur environnement physique et social des reflets (Abric, 1994). 
Elles sont des cadres de référence à partir desquels la réalité se construit dans un groupe social donné et elles constituent un guide pour l'action des membres de ce groupe (Guimelli, 1995). Elles sont créées à partir de nos interactions avec les autres et elles constituent la base de nos liens sociaux.

Pour connaître les représentations généralement acceptées quant au silence de participants dans différents types de groupes, nous avons relevé les interventions privilégiées dans les champs de l'animation des groupes de tâche, du travail social et du counseling ou de la psychothérapie.

La plupart des auteurs s'entendent pour dire que les silences ne peuvent tous être traités de la même manière dans un groupe. Être silencieux, c'est s'intéresser ou s'ennuyer, refuser ou consentir, bouder ou admirer, s'évader ou se concentrer. Selon Fages (1990), chaque silence demande une réponse appropriée, et c'est lorsqu'on ne considère qu'une seule interprétation qu'on risque de s'engager dans l'engrenage de la brouille. Heap (1994), Lewis (1977) et Ward (1974) ont reconnu et décrit une variété de silences qu'ils ont rencontrés dans leur pratique respective en travail social et en psychothérapie. La synthèse de leurs travaux permet de dégager au moins sept types de silence individuel (silence d'un seul membre) ou collectif (silence du groupe entier) :

1. le silence de transition permet aux personnes ou au groupe de se familiariser avec une situation nouvelle;

2. le silence d'intégration survient lorsqu'une émotion intense se manifeste ou à la suite d'une prise de conscience de quelque chose d'important;

3. le silence d'embarras se manifeste à la suite d'une révélation difficile, comme réaction à un choc ou dans une situation conflictuelle;

4. le silence de démission est une réaction à la pauvreté des interactions ou le résultat d'une évaluation négative du potentiel du groupe;

5. le silence de dépendance affective se manifeste devant un intervenant qu'on considère comme détenteur de l'autorité; 
6. le silence lié à l'appréhension de parler en public peut être un signe de timidité ou de manque d'assurance; il est souvent associé à un taux élevé d'anxiété;

7. le silence d'agressivité passive est un moyen utilisé à l'occasion d'une lutte de pouvoir (avec l'animateur ou entre les membres), une réaction de protestation contre des normes, des façons de faire ou des attitudes qui déplaisent, ou une manifestation d'hostilité envers l'organisme et la personne qui représente cet organisme; il provoque souvent un inconfort.

Malgré ce soin pris pour distinguer les différents types de silence et l'insistance récente mise par quelques auteurs sur le fait que ces silences ne doivent pas être systématiquement considérés d'une manière négative, il demeure que les tendances dominantes dans les milieux des services sociaux et de l'éducation incitent les intervenantes et les intervenants à interpréter le comportement des personnes silencieuses comme des problèmes à résoudre.

Cette tendance est sans doute liée à l'inconfort généralement ressenti face aux silences, inconfort indissociable des représentations négatives que les intervenantes et intervenants se font de ce mode d'expression. À ce propos, Ward (1974, p. 12) souligne que les sons du silence sont si difficiles à supporter que la plupart d'entre nous ne peuvent les tolérer tels qu'ils sont. Heap partage le même point de vue. Selon lui :

Peu de phénomènes de groupe soulèvent autant d'anxiété que le silence. II suffit de quelques secondes pour que nous commencions à nous inquiéter : "Qu'estce qui ne va pas? Vont-ils rester toujours en silence? " "Que puis-je faire pour le rompre? " et parfois "Je voudrais n'être jamais venu ici » (1994, p. 103).

Si l'on admet que le silence peut être positif, la plupart des auteurs s'attachent pourtant à proposer des interventions visant à remédier à la situation. "L'agenda caché » des intervenants est habituellement de faire en sorte que tous s'expriment de manière presque égale.

Quatre représentations dominantes du silence se dégagent des écrits sur le sujet: 1 . le silence comme signe de résistance, 2. le silence comme indice de souffrance psychologique, 3. le silence comme offense aux autres ou encore 4 . le silence comme 
réaction à l'oppression. Ces représentations du silence méritent d'être examinées d'un peu plus près, parce qu'elles conditionnent les émotions des intervenantes et intervenants vis-à-vis de ce phénomène et qu'elles orientent leur conduite.

\section{Le silence comme signe de résistance}

C'est surtout en counseling de groupe et en psychothérapie que le silence est présenté comme une résistance, c'est-à-dire comme une attitude qui fait partie intégrante de l'approche défensive de la personne face à la vie, un moyen qu'elle a trouvé pour éviter l'anxiété suscitée par l'éventualité d'une rencontre engageante avec les autres (Corey, 1981, p. 74). Selon cette représentation, le participant qui garde le silence dans un groupe est habité par un ensemble de peurs fondamentales, telles la peur de l'intimité, la peur de se révéler ou d'être démasqué par les autres, la peur d'être rejeté ou de n'avoir rien de valable à apporter, la peur d'ouvrir une brèche dans la muraille qui semble contenir un monde intérieur fragile et chaotique, la peur de devoir faire face à des aspects de soi qui chambarderont sa vie quotidienne, etc.

Si l'on reconnaît le silence comme un phénomène normal et tout à fait naturel de la dynamique des groupes, on l'interprète néanmoins comme une résistance à laquelle les responsables des groupes doivent se préparer à réagir. La logique de cette conduite se fonde sur le postulat, discutable à plusieurs égards, selon lequel le participant silencieux est un membre qui cause problème d'abord pour lui-même, mais aussi pour les autres. Selon Yalom (1975), les personnes qui bénéficient le plus des groupes sont celles qui s'y engagent; et cet engagement se traduit concrètement par l'expression verbale. Les membres silencieux profiteraient donc rarement des bénéfices du groupe. Le membre silencieux est source de problème pour les autres participants et pour le leader. À ce sujet, Corey souligne (1981) que les personnes qui disent presque toujours « Je n'ai à peu près rien à dire » ou " Je peux en apprendre autant en écoutant les autres qu'en parlant tout le temps » ont un impact négatif sur le groupe.

La compréhension et le traitement de telles résistances comptent parmi les principales raisons d'être du counseling et de la psychothérapie en groupe. Pour prévenir ou lever cette 
prétendue résistance, une pratique de plus en plus répandue consiste à établir « un contrat de groupe » qui explicite les normes que les membres doivent s'engager à respecter pour favoriser l'établissement d'un climat de confiance, le développement des personnes et la cohésion du groupe. Parmi ces normes, on retrouve très fréquemment un appel à la participation active des membres, un appel qui recouvre une invitation pressante à parler.

La stratégie d'intervention généralement préconisée pour lever cette supposée résistance consiste à inviter la personne silencieuse à exprimer ce qu'elle ressent dans son silence et à examiner l'effet qu'elle produit chez les autres. On suggère aussi au leader de s'engager lui-même à révéler ce qu'il éprouve dans la situation (Corey, 1981). Une autre intervention, plus radicale celle-là, consiste à demander aux autres membres du groupe comment ils interprètent le silence particulier d'une personne et comment ils y réagissent (Mucchielli, 1992).

\section{Le silence comme indice de souffrance psychologique}

En adhérant à cette représentation du silence, on considère très fréquemment les personnes silencieuses comme des personnes timides qui doivent être protégées, puisqu'elles souffrent énormément de leur silence. Loin de choisir de demeurer silencieuses, ces personnes subissent plutôt les effets pénibles et gênants de leur peur d'avoir à communiquer avec les autres. Les personnes qui sont affectées par cette peur, que Lederman (1982) nomme "l'appréhension de communiquer », sont presque constamment envahies par un sentiment d'anxiété lorsqu'elles anticipent une situation où elles devront communiquer. Cette appréhension ne se limite pas aux situations formelles qui exigent de parler en public. Elle est ancrée beaucoup plus profondément dans des fantasmes terrifiants qui incitent les personnes à craindre et à éviter toute situation potentielle d'interaction avec les autres, et cela, dans plusieurs sphères de leur vie. Le retrait qui s'ensuit fait que les personnes développent une image négative d'elles-mêmes, image d'autant plus difficile à corriger qu'elle est alimentée par les préjugés et par les attitudes condescendantes qu'elles subissent de la part des autres.

Un type d'intervention qui a longtemps été utilisé pour traiter cette problématique était " the speak up approach » (Lederman, 1982). La personne devait parler, qu'elle ait peur ou non de le 
faire. On croyait alors que le seul fait de parler ferait disparaître la peur (comme le fait de lancer en eau profonde une personne qui ne sait pas nager ferait disparaître la peur de l'eau). Si ce type d'intervention peut fonctionner pour des personnes légèrement affectées par l'appréhension de communiquer, il peut être totalement traumatisant pour d'autres qui en souffrent plus sévèrement. On a donc dû repenser cette méthode et on en a identifié d'autres qui pourraient être plus efficaces dans certains cas, comme l'hypnose, la désensibilisation systématique, la relaxation induite par le " biofeedback ", la participation à des groupes de counseling et la restructuration cognitive. Lederman (1982) mentionne que, même si « the speak up approach » est souvent valorisée en situation de groupe, c'est une méthode dangereuse à utiliser avec les personnes qui sont silencieuses, parce que cela peut renforcer leur grande peur de communiquer verbalement avec les autres.

D'autres auteurs, comme Grzybowski (1976), se sont employés à trouver des "recettes » pour intégrer différents types de personnes qui souffrent de leur silence. II décrit, entre autres, le "complexé » qui a l'impression d'être inférieur aux autres. Selon lui, il convient de tenter de l'intégrer en s'intéressant à ce qu'il fait et en l'interrogeant, mais en évitant le ton paternaliste. II y a aussi le «timide » qui n'est pas assez fonceur pour intervenir dans le groupe. II faudrait intervenir délicatement avec lui, afin de le mettre peu à peu en confiance dans le groupe.

Selon cette représentation du phénomène, les personnes silencieuses vivent beaucoup d'inconfort et de solitude (Ward, 1974) et nous devons, sans les brusquer, leur tendre des perches afin de les sortir de cet état qui les fait énormément souffrir (Lederman, 1982; Grzybowski, 1976; Ward, 1974).

On comprendra que cette représentation répandue du silence, si fondée soit-elle, peut inciter les intervenants à adopter une attitude exagérément complaisante envers les personnes silencieuses et, conséquemment, renforcer leur nervosité et leur sentiment d'inaptitude.

\section{Le silence comme une offense aux autres}

Souvent, le fait que certaines personnes ne participent pas à une conversation dans un groupe produit de l'inconfort chez les 
autres, leur silence étant alors interprété comme un signe de fermeture et d'hostilité, comme l'expression d'une agressivité passive. Dans la vie de tous les jours, on entend parfois dire que les personnes qui restent silencieuses en société et qui se préoccupent peu d'alimenter la conversation manquent d'un savoir-vivre élémentaire.

Cette représentation se manifeste aussi dans les milieux de pratique professionnelle auprès des groupes. Heap souligne, à ce propos, que: "Jusqu'à ces dernières années, on enseignait aux travailleurs sociaux et aux thérapeutes que le silence équivalait à de l'hostilité » (1994, p. 103). Ce genre d'enseignement n'est sans doute pas étranger au malaise éprouvé par les intervenants face au silence et à leur promptitude à intervenir.

Pourtant, au-delà de cet enseignement, Heap ajoute que les silences laissent aussi une place que plusieurs intervenants ont tendance à meubler de leurs peurs, de leurs sentiments d'incompétence et de leurs fantasmes. Quelques secondes de silence suffisent alors à les déstabiliser. Ils cultivent l'impression désespérante que le silence du groupe est dû au fait que ce qu'ils proposent n'intéresse pas les membres du groupe ou encore que les membres silencieux sont en colère, qu'ils sont entêtés ou qu'ils veulent exercer leur pouvoir contre eux. Exagérément centrés sur leur propre performance, ils paniquent et doutent de leur compétence (Heap, 1994; Ward, 1974; Lewis, 1977), plutôt que de chercher à comprendre ce qui se passe.

Si les silences persistent et se répètent, plusieurs intervenants habités par ces sentiments en viennent à chercher désespérément une façon de désamorcer une situation perçue comme menaçante pour leur propre image. Leur réaction spontanée pourra être de questionner indûment les personnes silencieuses pour trouver un exutoire à leur propre agressivité. Les intervenants pourront aussi s'employer eux-mêmes à libérer l'anxiété qui les tenaille ou chercher à gagner les faveurs du groupe par toutes sortes de plaisanteries, de compromis ou de minauderies. On imagine bien que ces tentatives sont, la plupart du temps, aussi inefficaces que dérisoires.

Les personnes silencieuses ne dérangent pas seulement les intervenantes et les intervenants. Dans un groupe, les autres participants se plaignent de leur conduite parce que ces personnes n'amènent pas d'eau au moulin et contribuent moins au 
progrès du groupe (Lederman, 1982). Leur attitude est frustrante pour les autres qui prennent le risque de s'exposer et qui aimeraient connaître l'opinion ou la réaction de ces êtres silencieux qui les observent (Boisvert, Cossette et Poisson, 1995). Eagan (1976) dira que le silence, qu'il soit intentionnel ou non, est un comportement manipulateur et contre-productif. Si les personnes silencieuses, admet-il, peuvent apprendre du groupe sans parler, elles n'en violent pas moins le contrat collectif en privant les autres de leur contribution. Elles brisent le contrat initial convenu avec le groupe et les responsables de l'intervention.

Dans cet esprit, les individus silencieux sont souvent associés au type " grand seigneur » ou encore au type " renfrogné » décrits par Grzybowski (1976). Le "grand seigneur » se croit supérieur aux autres et son silence signifie qu'il ne veut pas être dans le groupe. Le "renfrogné " est en désaccord avec l'existence même du groupe ou avec tout ce qui s'y passe. II adopte une attitude agressive et cynique en assistant passivement aux efforts des autres pour faire progresser le groupe, mais sans s'engager lui-même (Corey, 1981). Selon plusieurs auteurs, ces types de participants ne sont pas à leur place dans le groupe. Il faudrait donc absolument que le responsable du groupe intervienne pour les amener à participer, que ce soit en les faisant sourire ou par la confrontation, en tentant de les intéresser ou en les interrogeant jusqu'à ce qu'ils cessent de bouder. Si ces interventions légères ne suffisent pas, le responsable peut demander de rencontrer le membre récalcitrant à l'extérieur du groupe pour renégocier avec lui les conditions de sa participation.

\section{Le silence comme réaction à l'oppression}

Une quatrième représentation dominante du silence consiste à l'associer à une situation d'oppression. Dans certains cas, le silence d'un individu, d'un sous-groupe ou du groupe entier exprime un sentiment d'infériorité ou d'incompétence envers d'autres membres, perçus comme plus qualifiés, ou envers l'intervenant, considéré comme détenteur de la vérité. Dans d'autres cas, notamment dans des groupes d'entraide ou de counseling auprès de populations marginales, le silence peut aussi être le moyen de masquer un sentiment de honte ou de culpabilité par rapport à sa situation et une crainte d'être jugé (Heap, 1994, p. 107). Selon Batsleer (1994), la peur de parler s'explique par 
l'accumulation d'expériences négatives auxquelles certaines personnes ont été soumises lorsqu'elles ont tenté d'exprimer ce qu'elles vivaient. À la longue et à force d'être invalidées par le jugement des autres, les personnes qui occupent des positions marginales ou jugées inférieures dans la hiérarchie sociale apprennent à se méfier et à se taire.

Plusieurs observations empiriques permettent de constater que l'appropriation du temps de parole est effectivement liée au statut plus élevé du leader ou de certains membres, aux pouvoirs qu'ils détiennent et à leur position socialement dominante. Comme le démontre Bourdieu:

La compétence linguistique n'est pas une simple capacité technique, mais aussi une capacité statutaire. Cela signifie que toutes les formulations linguistiques ne sont pas également acceptables et que les locuteurs ne sont pas égaux (1992, p. 121).

Les personnes plus scolarisées ou placées plus haut dans une hiérarchie professionnelle parlent pour les moins scolarisées. Les personnes ayant de l'expérience parlent pour les personnes qui n'en ont pas. Les hommes parlent pour les femmes. Les personnes stigmatisées par leur marginalité (prestataires de la sécurité du revenu, homosexuels, personnes ayant éprouvé des problèmes de santé mentale, femmes ou enfants victimes de violence physique ou sexuelle, etc.) apprennent à cacher cette marginalité pour éviter de s'exposer aux préjugés. Dans ces contextes, le silence exprime à la fois une crainte d'être ignoré ou rejeté et l'existence d'une domination. II peut aussi refléter les habitudes de soumission des classes populaires devant la culture dominante des classes moyenne et supérieure (Heap, 1994).

Cette domination peut se vivre avec la conscience d'une certaine iniquité de départ et avec un sentiment de révolte retenue. Elle peut alors se traduire par le refus des personnes silencieuses de participer à un discours qui leur est étranger (voire hostile) ou par leur refus de donner la réplique attendue d'eux dans un langage qui n'est pas le leur et qu'on leur impose. Mais elle peut aussi se vivre d'une façon encore plus dévastatrice, lorsque les personnes silencieuses se taisent parce qu'elles en viennent à penser que les autres savent mieux qu'elles-mêmes ce qu'elles ressentent ou ce qu'elles devraient exprimer. Comme l'écrivait Bourdieu, « les classes dominées ne parlent pas, elles 
sont parlées » (1977, p. 4). Lorsque la révolte fait place à la résignation, les personnes opprimées se cantonnent dans le silence parce qu'elles ont été graduellement dépossédées de leur pouvoir de parler en leur nom. Elles en viennent à adhérer aux idéologies dominantes, à se conformer à des normes qui leur sont étrangères et à craindre leur propre liberté. Elles ne sont plus capables de se faire leur propre opinion, de prendre des décisions, de faire des choix en accord avec leur culture et leur réalité.

Devant ces formes de silence, Heap (1994) propose aux intervenants de commenter ce qu'ils comprennent, en soulignant la nécessité de renforcer l'estime de soi des membres, de valoriser leurs succès et capacités et de répondre positivement à leur initiative. II propose aussi de susciter les interactions en favorisant le développement de la solidarité dans le groupe. Batsleer (1994) ajoute que l'intervenant n'a pas à interpréter le silence d'un point de vue neutre et objectif. Au contraire, ce n'est qu'à partir de la reconnaissance de son propre engagement, de sa différence, de ses attentes cachées et de ses peurs que l'intervenant pourra faire des liens avec les messages exprimés par les silences. L'intervenant n'a pas non plus à se présenter comme le détenteur d'une quelconque connaissance supérieure lui permettant de décoder avec assurance les silences. Sa compréhension de ces silences ne peut être celle d'un expert qui comprendrait le sens de ce langage universel, sorte d'espéranto de la dynamique des groupes, qui transcenderait les histoires personnelles, les cultures, les différences de classes et les conflits.

\section{LES EFFETS PERVERS}

Les quatre représentations dominantes du silence exposées cidessus ont amené plusieurs auteurs à proposer une panoplie de stratégies d'intervention différenciées selon l'interprétation qu'on se fait des silences. Les enseignements traditionnels à ce propos considéraient toutes les formes de silence comme des signes de résistance, de manipulation ou d'agressivité passive. Sans réfuter tout à fait ces enseignements, on admet aujourd'hui que les motivations et les processus qui sous-tendent les silences des individus et des groupes sont infiniment plus complexes et variés. Ce qui se dégage des travaux récents, c'est 
d'abord l'importance d'être attentif aux différents sens des silences, puis de reconnaître qu'il n'y a pas de stratégie universelle qui serait applicable à toutes les situations et invariablement. Malgré ces nuances essentielles, on remarque toutefois que la tendance à considérer les silences comme des problèmes à résoudre reste très marquée et que les stratégies les plus largement préconisées se ramènent le plus souvent à interroger les personnes silencieuses sur la raison de leur mutisme.

Si ce souci d'équilibrer la participation, manifesté par les théoriciens et les praticiens, est des plus légitimes (Bertcher, 1994) et siles stratégies que ces derniers développent se révèlent utiles dans plusieurs situations, on doit toutefois savoir que ces stratégies employées envers les personnes silencieuses peuvent parfois causer plus de torts que de bienfaits ${ }^{3}$. II peut arriver en effet que les meilleures intentions du monde et que les pistes d'intervention apparemment les plus logiques provoquent des effets tout à fait contraires à ceux recherchés.

Interroger les personnes silencieuses, leur tendre gentiment des perches pour qu'elles s'expriment, utiliser la confrontation pour les amener à s'impliquer ou tenter de donner un sens à leur comportement sont quelques-unes des stratégies préconisées, mais qui conduisent fréquemment à accentuer le problème. Dans les termes de la théorie de la communication (Watzlawick, HelmickBeavin et Jackson, 1975) et du changement thérapeutique de l'école de Palo Alto (Watzlawick, Weakland et Fisch, 1975; Fisch, Weakland et Segal, 1986), on peut constater que ces interventions qui visent, au départ, à résoudre une difficulté mineure peuvent vite transformer cette difficulté en problème sérieux, à partir du moment où l'intervenant ou d'autres membres du groupe s'acharnent à appliquer toujours la même solution sans examiner ses effets. Voici trois situations, parmi d'autres, qui peuvent illustrer notre propos.

- Dans une première situation, les efforts de l'intervenant ou de certains membres du groupe pour donner à la personne silencieuse une place qu'elle ne prend pas amèneront celle-ci à devenir exagérément consciente du regard que les autres posent sur elle et à se soucier de leur donner la réplique appropriée.

3. À ce propos, Lederman souligne qu'il peut être plus néfaste de demander à un membre silencieux de s'exprimer que de demander à un membre qui s'exprime beaucoup de demeurer silencieux (1982, p. 280). 
Si la personne ciblée est initialement à l'aise dans son silence, notamment lorsqu'elle écoute les autres, lorsqu'elle se tait parce qu'elle n'a rien à ajouter à ce qui a été dit ou lorsqu'elle prend le temps d'apprivoiser une nouvelle situation, elle pourra commencer à se sentir moins à l'aise quand elle sentira le regard des autres porté sur elle. Délogée d'une situation relativement satisfaisante, elle développera une conscience exagérée d'ellemême ${ }^{4}$; elle apprendra que sa conduite n'est pas convenable pour les autres et commencera à se sentir inadéquate. Elle pourra alors devenir exagérément nerveuse à l'idée de devoir parler à un moment qu'elle n'aura pas choisi. Afin d'éviter d'attirer davantage l'attention sur elle, elle aura tendance à donner des réponses de conformité, ce qui pourra faire croire aux autres qu'elle manque d'engagement et d'authenticité et ce qui pourra confirmer l'hypothèse du silence comme manifestation d'une résistance. Par ailleurs, plus la personne silencieuse cherchera à émerger de l'impasse, plus elle se repliera sur elle-même par crainte de ne pas donner la réplique conforme aux attentes des autres.

- Une deuxième situation, semblable, survient lorsque se manifestent des attitudes de condescendance de la part de l'intervenant ou des membres du groupe, qui agissent envers les personnes silencieuses comme si elles n'étaient que faibles ou vulnérables. Pour se « mettre à leur niveau » (comme s'il s'agissait d'un niveau inférieur), on utilisera un langage rudimentaire, on tentera de leur demander leur avis sur des sujets très simples, on leur tendra des perches à propos de tout et de rien, etc. Dans une enquête à ce propos, Lederman (1982) rapporte qu'un très grand nombre de personnes silencieuses témoignent du

4. Cette «conscience de soi », traduction du phénomène appelé «selfconsciousness", est la conscience qu'on a de la présence des autres qui nous regardent et qui peuvent nous juger. Cette seule condition suffit généralement à déclencher un mécanisme d'auto-centration qui influence sensiblement la conduite de la personne qui se sent observée. En situation de groupe, cette conscience de soi peut provoquer des malaises qui peuvent amener une personne à perdre ses moyens; elle dira alors qu'elle est gênée, intimidée, déstabilisée par la situation. C'est comme si le fait de sentir la pression du regard des autres empêchait d'accorder l'attention nécessaire à ce qu'on a à faire ou à dire : on est décentré. À lui seul, ce phénomène peut inhiber l'expression ou inciter les personnes à se conformer, simplement parce qu'elles ne souhaitent pas être remarquées. 
malaise qu'elles ressentent lorsqu'elles se voient étiquetées comme timides ou démunies, compte tenu de leur statut ou de certaines autres de leurs caractéristiques. Que leur silence s'explique effectivement par de la timidité, par une situation réelle d'oppression ou qu'il s'explique tout simplement par une préférence à écouter ne change pas fondamentalement les effets d'une attitude condescendante sur elles. Ce traitement exagérément bienveillant contribue plutôt à les enfermer dans une situation d'infériorité; il risque de semer le doute en elles quant à leurs forces et à leurs ressources. Les personnes silencieuses finissent par considérer qu'elles doivent parler pour réhabiliter leur image aux yeux des autres. Le phénomène de la prophétie autoréalisante se manifeste alors: le traitement particulier que la personne reçoit renforce son impression d'être "à part des autres » et, en retour, cette impression l'amène effectivement à " être à part ».

- Une autre réaction typique est celle de la personne qui commence par être agacée, puis vraiment agressive devant le harcèlement subtil qu'elle ressent de la part des autres qui veulent la faire parler. Cette réaction suit souvent l'une des deux situations précédentes. Plus l'intervenant ou d'autres membres du groupe s'acharnent à aider une personne à s'exprimer, plus cette dernière s'obstine dans son mutisme, finissant par croire que ceux et celles qui veulent tant la faire parler ne peuvent qu'être de mauvaise foi lorsqu'ils manifestent une telle cruauté mentale. En retour, plus la personne silencieuse se renferme pour se protéger, plus l'intervenant ou les autres membres du groupe croient qu'ils ont raison de la pousser dans ses derniers retranchements afin de l'amener à parler.

Dans ces trois situations, on observe une spirale relationnelle où les parties en présence s'enlisent inexorablement dans des positions complémentaires de plus en plus rigides et insatisfaisantes, en faisant chacune " plus de la même chose » pour tenter de résoudre les problèmes qu'elles ont créés. Watzlawick, Helmick-Beavin et Jackson (1979) remarquent que le problème typique des relations dites complémentaires surgit quand " $X$ » veut que " $Y$ » le considère d'une manière qui contredit la manière dont " $Y$ » cherche lui-même à être considéré par « $X$ », et vice versa. Ce qui est en jeu, ce n'est plus tant le comportement plus ou moins adapté de l'un et de l'autre, mais leur identité même qu'ils cherchent à affirmer et qui reste menacée tant que l'autre 
refuse de jouer le rôle complémentaire attendu. Dans le cas de la relation entre un silencieux et un intervenant qui cherche à le faire parler, on comprendra que le silencieux ne pourra se sentir confirmé dans la relation que si son comportement silencieux est respecté et bien compris, tandis que l'intervenant ne se sentira confirmé que si le silencieux accepte de lui exprimer verbalement les raisons de son silence. Si le silencieux n'obtient pas la reconnaissance de l'intervenant, il le jugera incompétent et insensible (ce que l'intervenant craint par-dessus tout); et si l'intervenant ne parvient pas à amener le silencieux à s'exprimer selon ses attentes, il le classera dans la catégorie des membres timides ou récalcitrants. On remarquera alors chez l'un des partenaires, ou chez les deux, un sentiment croissant de frustration (Watzlawick, Helmick-Beavin et Jackson, 1979, p. 107).

Ce sont aussi des situations typiques qui illustrent le paradoxe décrit par l'école de Palo Alto, où c'est dans la solution que réside le problème. Le jeu sans fin qui consiste à s'accrocher toujours à une même conduite, en rajoutant encore et encore, bien qu'on sache qu'elle n'a pas fonctionné auparavant, ne garantit pourtant pas qu'elle aura les effets recherchés après un certain nombre de tentatives. Au contraire, le problème est aggravé par les efforts mêmes que les partenaires déploient pour tenter de le résoudre en faisant toujours "plus de la même chose ». Plus l'intervenant insiste pour qu'un membre silencieux parle, plus ce dernier se sentira anxieux, agressif ou incompris et plus il se refermera sur lui-même. En outre, plus le membre silencieux se refermera sur lui-même, plus l'intervenant se sentira en droit d'insister. En réponse à ce type d'impasse, la perspective de Palo Alto propose de recadrer le problème, ce qui suppose une modification substantielle de ses représentations du réel et l'adoption d'une conduite radicalement différente.

Recadrer, c'est modifier le contexte conceptuel et/ou émotionnel d'une situation, ou le point de vue selon lequel elle est vécue, en la plaçant dans un autre cadre, qui correspond aussi bien, ou même mieux, aux "faits » de cette situation concrète, dont le sens, par conséquent, change complètement (Watzlawick, Weakland et Fisch, 1975, p. 116).

Une façon de recadrer une situation problématique consiste à se méfier des interventions logiques, à reconnaître qu'elles 
peuvent aggraver une difficulté et à envisager d'autres stratégies d'intervention même si elles peuvent paraître contraires au bon sens. Parmi ces stratégies qui peuvent créer une solution alternative, mentionnons l'injonction paradoxale et la prescription de symptôme, qui consistent à demander à l'autre de faire quelque chose de tout à fait contraire à ce qu'on voudrait qu'il fasse, tout en le plaçant dans une situation telle qu'il ne parviendra pas à le faire. Ici, il s'agirait de placer la personne silencieuse dans une situation où elle pourrait difficilement résister à son envie de parler, mais en la convainquant qu'elle doit s'interdire de parler ${ }^{5}$. Une autre façon de recadrer le problème est précisément de l'ignorer et d'agir avec la personne plus silencieuse comme on agirait avec une autre, notamment en lui demandant directement son avis ou ses réactions sur différents sujets. En plus d'éviter les effets paralysants des étiquettes qu'on met aux gens, cette stratégie a l'avantage de rompre le modèle d'interaction auquel la personne a pu finir par s'habituer; ces deux éléments combinés favorisent le développement de nouvelles dynamiques relationnelles à l'intérieur desquelles les choses peuvent se passer tout autrement.

\section{LES COÛTS ET LES BÉNÉFICES DU SILENCE}

Le réexamen d'une situation considérée comme problématique à la lumière de la théorie de l'échange peut permettre aux intervenants de reconsidérer leurs propres réactions ainsi que le sens qu'ils donnent aux comportements des membres du groupe face au silence. Selon la théorie de l'échange, le silence persiste tant que les partenaires en présence en retirent plus de bénéfices que ce qu'il leur en coûte ou tant qu'ils ne perçoivent pas une solution de rechange qui pourrait être plus satisfaisante. En d'autres mots, les partenaires d'un groupe peuvent se sentir mal à l'aise dans une situation, mais estimer qu'ils ne feraient qu'empirer la situation s'ils décidaient de modifier leur conduite mutuelle ou s'ils risquaient de changer radicalement de stratégie pour transformer leur rapport. En ce sens, Pépin mentionne que :

5. Pour réaliser une telle intervention, il faut d'abord que l'intervenant ait une bonne compréhension de la dynamique interactive et des efforts qui ont été faits pour changer la situation problématique (Fisch, Weakland et Segal, 1986). Souvent, toutes les stratégies qui ont été tentées dans le passé véhiculaient le même message. 
Nous avons tous (et toujours) de bonnes raisons d'agir comme nous le faisons, même si nous en doutons nousmêmes. II ne faut jamais oublier que nous agissons (et pensons) pour nous ajuster aux effets continuels de l'interaction sur nous (1993, p. 25).

Ainsi, le silence peut procurer aux personnes silencieuses plusieurs bénéfices différents et parfois conjugués: 1. se protéger en évitant de se dévoiler; 2 . éviter de se tromper ou d'être jugé; 3 . faire l'économie de toutes les énergies qu'il faut déployer pour se faire entendre ou pour se rendre utile et intéressant; 4. demeurer fidèle à ses habitus et à ses convictions quant à la place du silence et de la parole; 5 . se préserver de l'inconfort ou du déplaisir que provoquerait un dialogue avec des personnes qui ne cherchent qu'à avoir raison; 6 . jouir du malaise qu'on provoque chez elles en leur opposant son mutisme; 7 . savourer le simple plaisir qu'on éprouve à écouter. La question est donc de savoir si ces bénéfices seront supérieurs aux coûts à supporter, notamment celui d'avoir à subir les interventions persistantes des autres et celui de risquer d'être perçu négativement.

Quant aux membres du groupe plus loquaces, les bénéfices qu'ils retirent sont aussi variés : 1 . avoir plus de temps de parole; 2. avoir plus de pouvoir dans l'orientation des discussions et des actions du groupe; 3 . avoir une tribune et une audience pour se faire valoir; 4 . échapper à la nécessité d'avoir à composer avec des points de vue ou des propositions d'action qu'on cherche à éviter ou à étouffer; 5. pouvoir octroyer aux personnes silencieuses le rôle de boucs émissaires. En contrepartie, ils peuvent se sentir privés de la contribution des personnes silencieuses ou se sentir injustement jugés par elles, qui choisissent la position confortable de critiquer dans leur for intérieur sans se compromettre dans l'action.

Le responsable de groupe peut retirer ces mêmes bénéfices en créant une collusion subtile avec les personnes qui s'allient à lui pour réprouver plus ou moins directement la conduite des personnes silencieuses ${ }^{6}$. Ainsi, malgré le fondement rationnel

6. Parfois, on a tendance à accorder trop d'attention à des membres qui participent comme on le désire, à ceux qui s'expriment facilement, qui disent ce qu'on aime entendre et avec qui on a des affinités; c'est rassurant pour l'animateur de leur laisser de la place. On doit toutefois se 
qui amène l'intervenant à tout mettre en œuvre, du moins en apparence, pour équilibrer la participation, celui-ci a parfois un intérêt non avoué, mais néanmoins très puissant, à entretenir ou à aggraver le problème. Cela se produit, notamment, lorsque les personnes silencieuses risquent de faire part d'expériences ou de questions qui déstabiliseraient le groupe, lorsque leur parole risque d'ébranler la fragile pertinence du programme d'intervention proposé ou lorsque l'intervenant a le sentiment que les personnes silencieuses portent en elles une agressivité avec laquelle il ne saurait comment composer. En fait, quelle que soit la stratégie adoptée par l'intervenant pour inciter les personnes silencieuses à s'exprimer, celui-ci peut se demander s'il veut vraiment entendre ce qu'elles ont à exprimer ou s'il ne cherche pas plutôt à réduire sa propre anxiété, à se donner bonne conscience ou à chercher un moyen d'accroître son emprise sur le groupe.

\section{CONCLUSION}

À la suite de cette réflexion, on doit convenir qu'il y a certaines situations où le silence est problématique dans un groupe. Ainsi, il faut intervenir lorsque le silence exprime l'hostilité ou la contestation. On fait alors face à une agressivité passive dont le sens doit être élucidé. De la même manière, le silence qui cache un réflexe parasitaire ou un refus de certains membres de faire leur part du travail doit être démasqué et des solutions équitables doivent être trouvées. Dans ces situations, le silence n'est pourtant pas au cœur des problèmes : il couvre plutôt d'autres problématiques qui doivent être abordées directement.

Dans d'autres situations, le silence cause d'abord des problèmes aux membres du groupe "qui parlent ». II est alors utile de se demander si leur malaise ne traduit pas leur propre insécurité projetée, leur crainte de se faire juger, voire leur besoin de trouver un bouc émissaire. Afin d'éviter de tomber dans le piège qui consisterait à faire porter tous les problèmes par les personnes silencieuses, il faut prendre le temps de dégager les enjeux implicites du groupe.

Enfin, il faut savoir agir si la personne silencieuse se sent mal, si elle souffre de son propre silence en se repliant sur elle-même.

méfier de la sécurité qu'apportent les verbomoteurs qui réagissent toujours positivement à ce qu'on amène. 
La stratégie de l'ignorance et du laisser-faire pourrait en effet porter préjudice à la personne qui se sent prise de panique, impuissante ou résignée parce qu'elle ne sait pas comment se sortir de l'impasse dans laquelle elle se trouve. Laissée à ellemême, elle ne ferait que se désespérer davantage. L'intervention appropriée exigera toutefois une décristallisation des modèles interactifs dans lesquels s'est installé le silence. Cette décristallisation peut être favorisée par divers moyens qui ne pèsent pas trop lourdement sur la personne silencieuse, notamment: le travail en sous-groupe, la diversification des activités et des formes d'expression, la transformation de la disposition de l'espace, l'élaboration de manières de faire qui respectent sa culture, etc. À l'occasion, le responsable du groupe peut aussi agir comme porte-parole de la personne silencieuse. Avec la permission explicite ou implicite de cette dernière, il interprétera ce qu'il comprend d'elle et l'invitera à le corriger si elle le juge nécessaire. En entendant parler d'elle et en voyant les autres membres du groupe porter intérêt à ce qui est dit, la personne silencieuse ne tarde généralement pas à apporter des nuances, à corriger certains propos et à s'engager dans la discussion à un rythme qui lui convient. Cette manière de faire assure une place à la personne silencieuse et facilite sa participation.

Au-delà des questions qui touchent l'efficacité ou la finalité des stratégies d'intervention visant à favoriser l'expression des personnes silencieuses, cet article soutient l'idée qu'il ne faut pas parler à tout prix pour s'exprimer dans un groupe. Aussitôt que deux personnes ont conscience de leur présence réciproque, elles communiquent. Afin de bien mesurer la portée du premier axiome de la communication de l'école de Palo Alto qui dit que "On ne peut pas ne pas communiquer » (Watzlawick, Beavin et Jackson, 1979, p. 46), il convient surtout de s'exercer à survivre à quelques silences et d'apprivoiser diverses autres formes d'expression que la parole.

Reconnaître que la communication est un processus à multiples canaux et que le langage verbal n'est qu'un de ces canaux permet de se sentir en communication autant avec les membres silencieux d'un groupe qu'avec les personnes qui s'expriment davantage verbalement. Un regard complice, un sourire, une réponse à une expression non verbale, la planification d'activités de groupe qui se fondent sur d'autres canaux de communication 
que le langage verbal sont quelques moyens parmi bien d'autres que la personne qui anime peut utiliser pour échanger des vues avec les membres silencieux du groupe. Dans cette perspective, l'intervenant de groupe peut remettre en question les représentations négatives qu'il se fait du silence en y voyant d'abord un mode riche et irremplaçable d'expression. Ce changement de perspective, s'il ne résout pas tous les problèmes, a au moins le mérite de ne pas en créer là où il serait possible de s'entendre sans nécessairement parler, parler et parler.

Chantal LECLERC

Professeure

Département d'orientation, d'administration et d'évaluation en éducation Faculté des sciences de l'éducation Université Laval

Patricia PAQUET

Conseillère d'orientation Cégep Beauce-Appalaches 


\section{Références bibliographiques}

ABRIC, J.-C. (1994). Pratiques sociales et représentations, Paris, PUF.

BANDURA, A. (1980). L'apprentissage social, Bruxelles, Pierre Mardaga.

BATSLEER, J. (1994). "Silence in working across difference: Groupwork in a youth and community work training context », Groupwork, vol. 7, n³, p. 197-209.

BERTCHER, H.J. (1994). Group participation, Techniques for leadership and members, Londres, Sage.

BILMES, J. (1994). "Constituting silence: Life in the world of total meaning ", Semiotica, vol. 98, $n^{\circ} 1$, p. 73-87.

BOISVERT, D., F. COSSETTE et M. POISSON (1995). Animation de groupes, Cap-Rouge, Québec, Presses interuniversitaires.

BOURDIEU, P. (1992). Réponses, avec Loïc J.D. Wackuant, Paris, Seuil.

BOURDIEU, P. (1977). "Une classe objet », Actes de la recherche en sciences sociales, $n^{\text {os }} 17-18$, p. 2-5.

CHING, W. et S.S. PROSEN (1980). "Asian-Americans in Group Counseling: A case of Cultural Dissonance », Journal for Specialists in Group Work, novembre, p. 228-232.

COLLIER, L.C. (1993). Teacher's tales: teaching native students, John Abbott College, Sainte-Anne-de-Bellevue, Québec.

COREY, G. (1981). Theory and practice of group counseling, California, Brooks/Cole Publishing Company.

EAGAN, G. (1976). Interpersonal Living : A Skills/Contract Approach to Human Relations Training in Groups, California, Brooks/ Cole Publishing Company.

FAGES, J.-B. (1990). Communiquer entre personnes en groupe, Paris, Éditions Privat.

FISCH, R., J.H. WEAKLAND et L. SEGAL (1986). Tactiques $d u$ changement: Thérapie et temps court, Paris, Seuil.

FREIRE, P. (1974). Pédagogie des opprimés, Paris, Librairie François Maspero.

GRZYBOWSKI, G. (1976). "Comment réussir une réunion », Psychologie, $\mathrm{n}^{\circ} 81$. 
GUIMELLI, C. (1995). "L'étude des représentations sociales ", Psychologie française, $\mathrm{n}^{\circ}$ 40-4, p. 367-374.

GUIMOND, S. (1994). "Les groupes sociaux », dans R.J. Vallerand (dir.), Les fondements de la psychologie sociale, Montréal, Gaëtan Morin, p. 655-705.

HALL, K. (1984). La danse de la vie, Paris, Seuil.

HEAP, K. (1994). La pratique du travail social avec les groupes, Paris, ESF éditeur.

HIGGS, J. A. (1992). "Working with groups : Strategies for effective group », The Journal for Specialists in Group Work, vol. 17, $n^{\circ} 2$, p. 67-73.

LEDERMAN, L. (1982). "Suffering in Silence : The effects of fear of talking on small group participation », Group and Organization Studies, Sage Publications Inc., vol. $7, n^{\circ} 3$, p. 279294.

LEHTONEN, J. et K. SAJAVAARA (1985). "The silent Finn», dans D. Tannen et M. Saville-Troike " (dir.), Perspectives on Silence, Norwood, NJ, Ablex.

LEWIS, B.F. (1977). "Group silences », Small Group Behavior, vol. $8, n^{\circ} 1$, p. 109-120.

MARC, E. et D. PICARD (1989). L'interaction sociale, Paris, PUF.

MONTEIL, J.-M. et L. MAILHOT (1988). "Éléments d'une représentation sociale de la formation: Analyse d'une enquête auprès d'une population de formateurs », Connexions, vol. 51, p. 9-26.

MUCCHIELLI, R. (1992). La conduite de réunions (13 éd.). Paris, ESF éd., Coll. Formation permanente en sciences humaines.

PÉPIN, Y. (1993). Intervention psycho-sociale, Recueil de textes, Département de counseling et orientation, Université Laval.

RICHARD, B. (1995). Psychologie des groupes restreints, Québec, Presses interuniversitaires.

SFEZ, L. (1992). La communication ( $1^{\text {re }}$ édition : 1991), Paris, PUF.

WARD, J.T. (1974). "The Sounds of Silence: Group Psychotherapy with Non-Verbal Patients », Perspectives-in-PsychiatricCare, vol. 12, n 1, p. 13-19.

WATZLAWICK, P., J. HELMICK-BEAVIN et D.D. JACKSON (1979). Une logique de la communication, Paris, Seuil.

WATZLAWICK, P. (1991). Les cheveux du baron de Münchhausen, Paris, Seuil. 
WATZLAWICK, P., J. WEAKLAND et R. FISCH (1975). Changements, paradoxes et psychothérapie, Paris, Seuil.

YALOM, I.D. (1975). The Theory and Practice of Group Psychotherapy $\left(2^{\mathrm{e}}\right.$ éd.), New York, Basic Books.

YAMAGUCHI, T. (1986). "Group Psychotherapy in Japan Today ", The International Journal of Psychotherapy, Madison, International Universities Press Inc., vol. 36, $n^{\circ} 4$, p. 567-578. 